\title{
A Proof-Theoretic Semantics for Transitive Verbs with an Implicit Object
}

\author{
Nissim Francez \\ Computer Science faculty, Technion \\ Haifa, Israel \\ francez@cs.technion.ac.il
}

\begin{abstract}
The paper presents a proof-theoretic semantics for sentences headed by transitive verbs allowing an unexpressed (implicit) object. Such sentences are shown to have the same (proof-theoretic) meaning as the same sentences with an explicit existentially quantified object something.

This semantics is contrasted with a model-theoretic semantics based on truthconditions in models. The models used contain in their domain "filler" elements, that have an unclear extra-theoretic significance with an unclear ontological commitments. In contrast, the proof-theoretic meaning is appealing to formal (syntactic) resources that carry no ontological commitment.

Furthermore, the sameness of meaning is based on sameness of deductive role within a meaning-conferring proofsystem, based on use.
\end{abstract}

\section{Introduction}

The purpose of this paper is to provide yet another argument for the benefits of proof-theoretic semantics (PTS), when applied to natural language $(\mathrm{NL})$, over the traditional model-theoretic semantics (MTS), by focusing on what came be known as unexpressed objects of transitive verbs (also referred to as implicit arguments) as manifested, for example, by an intransitive use of a transitive verb, as in $^{1}(1(\mathrm{i}))$ and(1(ii)) below.

$$
\text { (i) John ate an apple (ii) John ate }
$$

\footnotetext{
${ }^{1}$ All natural language expressions used in examples are displayed in the San Serif font, and are always mentioned, never used.
}

PTS is a theory of meaning serving as an alternative to the more traditional Model-Theoretic Semantics (MTS). While the latter identifies meaning with truth-conditions (in arbitrary models of a suitable form), the former identifies meaning with canonical derivability conditions in a meaningconferring proof system. Those canonical derivability conditions provide grounds for assertion. For a presentation of the motivation for PTS and a discussion of its advantages over MTS, the reader is referred to the Introduction sections of Francez and Dyckhoff (2010) or Francez and Ben-Avi (2015), which present a PTS for extensive fragments of English. A full presentation can be found in Francez (2015b).

Turning to the unexpressed arguments, the essence of the MTS-based meaning is to interpret such arguments as existentially quantified. Hence, (1(ii)) above is seen as equivalent to

$$
\text { John ate something }
$$

with meaning expressed as usual by a (simplytyped) $\lambda$-term, here expressible in 1st-order logic (using simple types on variables and constants):

$$
\exists x^{e} \text {.eat }^{(e,(e, t))}(x, \text { john })
$$

In Dowty (1982), (1(ii)) is seen as resulting from (1(i)) by a general procedure of argument reduction.

The structure of the rest of the paper is as follows. In Section 2 I briefly survey two recent approaches to obtaining the above mentioned truthconditions (MTS-meaning). In Section 3 I briefly review the PTS of a minimal fragment of English in which the issue of unexpressed arguments arises. Fuller details about the PTS (for larger fragments) can be found in Francez and Dyckhoff (2010) or Francez and Ben-Avi (2015) and in part II of Francez (2015b). Section 4 presents the the 
PTS account of the unexpressed argument issue, and compares it to the reviewed MTS accounts. Section 5 ends with some conclusions.

\section{A brief review of some model-theoretic approaches}

The topic of unexpressed argument has a long history. I will mainly relate to Blom et al. (2012) (see there for references to previous work), and to Giorgolo and Asudeh (2012).

The common feature of those treatments of the unexpressed argument is imposing the sameness of meaning of an intransitive verb used with an implicit argument with the meaning of that verb used transitively with an existentially quantified object. For example,

$$
\llbracket \text { Mary ate } \rrbracket=\exists x \cdot \llbracket \text { Mary ate } x \rrbracket
$$

The difference between Blom et al. (2012) and Giorgolo and Asudeh (2012) is the formalism used for composing meanings, a difference immaterial for my purpose. I consider here only the issue of meaning of such constructs, and not with the syntax-semantics interface used to derive those meanings compositionally, so I will take Blom et al. (2012) as the representative of the MTS to implicit arguments.. A completely different model-theoretic approach is presented in Carlson (1984), appealing to models with (Neo Davidsonian) events and event modifiers. See Blom et al. (2012) for a discussion of this kind of MTS.

The main features of the proposed analysis are the following (expressed in a somewhat different notation).

1. The standard domains $D_{\tau}$ of interpretation of types $\tau$ in (simply-typed) $\lambda$-calculi are extended with extra elements, designated generically as ' $*_{\tau}$ ', called the filler value of the domain of $\tau$. The type-system is extended so that for every type $\tau$ there is a corresponding extended type $\tau^{\varnothing}$ accommodating the default value so that $D_{\tau^{\varnothing}}=D_{\tau} \cup\left\{*_{\tau}\right\}$ (where the union is disjoint).

See below the qualms about this solution.

2. The lexical meaning of verbs licensing unexpressed objects is expressed via a function of the (notationally slightly modified) form

$$
\begin{array}{r}
\operatorname{option}\left(x_{\tau_{1}}^{o}, F_{\left(\tau_{1}, \tau_{2}\right)}, d_{\tau_{2}}\right) \\
\quad={ }^{d f .} \begin{cases}d & x=*_{\tau_{1}} \\
F(x) & \text { otherwise }\end{cases}
\end{array}
$$

Here $d_{\tau_{2}}$ is the default value of type $\tau_{2}$, chosen according to a global strategy: an existential closure of the word in the lexical semantic value of which this particular use of option is embedded. The option operator is of type $\tau_{1}^{o} \times\left(\tau_{1}, \tau_{2}\right) \times \tau_{2}$. The function $\lambda x$.option $(x, F, d)$ is therefore of type $\left(\tau_{1}^{o}, \tau_{2}\right)$.

Example 2.1 Consider the lexical semantic value associated ${ }^{2}$ with the verb ate, assuming it licensed an implicit object.

$$
\begin{gathered}
y_{e^{o}}, \\
\lambda y_{e^{o}} \lambda x_{e} \cdot \operatorname{option}\left(\lambda u_{e} \cdot \text { ate }_{(e,(e, t))}\left(u_{e}\right)\left(y_{e}\right),\right. \\
\left.\exists x_{e} \cdot \text { ate }(x)(y)\right)
\end{gathered}
$$

Now consider the two cases for ate.

explicit object: Mary ate an apple

See Figure 1:

\section{implicit object: Mary ate}

See Figure 2:

The extra-theoretic interpretation of such "filler elements" $*_{\tau} \in D_{\tau^{o}}$ is non-obvious, as is the presence of such elements in lexical meaning assignments of NL words. They cary an ontological commitment which is left unexplained.

As an example of one problematic issue involving filler elements, consider selectional restrictions. Typically, the are ignored, but there are ways of incorporating them into the type-based analysis above. Suppose one wants to admit

$$
\text { Mary ate an apple }
$$

but to exclude

\section{Mary ate a chair}

Both are admissible by the above analysis. One way of imposing selectional restrictions is by refining the type $e$, to include a sub-type $e_{\text {addible }}$,

\footnotetext{
${ }^{2}$ I ignore here the finer points involved in implementing this construction in abstract categorial grammar, orthogonal to my main concern.
} 


$$
\begin{aligned}
& \lambda y_{e^{o}} \lambda x_{e} \cdot \mathbf{o p t i o n}\left(y_{e^{o}}, \lambda u_{e} \cdot \text { ate }_{(e,(e, t))}\left(u_{e}\right)\left(y_{e}\right), \exists x_{e} \cdot \text { ate }_{(e,(e, t)}(x)(y)\right)(\llbracket \text { an apple } \rrbracket)(\llbracket M a r y \rrbracket) \\
& =\lambda x_{e} \cdot \text { option }\left(\llbracket \text { an apple } \rrbracket, \lambda u_{e} \cdot \text { ate }_{(e,(e, t))}\left(u_{e}\right)\left(y_{e}\right)(\llbracket \text { an apple } \rrbracket)(\llbracket \text { Mary } \rrbracket), \exists x_{e} \cdot \text { ate }_{(e,(e, t))}(x)(y)\right) \\
& =\lambda u \cdot \text { ate }(u)(\llbracket \text { an apple } \rrbracket)(\llbracket M a r y \rrbracket) \\
& =\text { ate }(\llbracket \text { an apple } \rrbracket)(\llbracket M a r y \rrbracket)
\end{aligned}
$$

The second step is due to $\llbracket$ an apple $\neq *_{e}$.

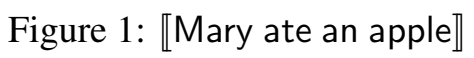

$$
\begin{aligned}
& \left.\lambda y_{e^{o}} \lambda x_{e} \cdot \mathbf{o p t i o n}\left(y_{e^{o}}, \lambda u_{e} \cdot \text { ate }_{(e,(e, t))}\left(u_{e}\right)\left(y_{e}\right), \exists x_{e} \cdot \text { ate }_{(e,(e, t)}(x)(y)\right)\right)\left(*_{e}\right)(\llbracket \text { Mary } \rrbracket) \\
& =\lambda x_{e} \cdot \text { option }\left(*_{e}, \lambda u_{e} \cdot \text { ate }_{(e,(e, t))}\left(u_{e}\right)\left(*_{e}\right)(\llbracket \text { Mary } \rrbracket), \exists x_{e} \cdot \text { ate }(x)(y)\right)(\llbracket \text { Mary } \rrbracket) \\
& =\exists x . \text { ate }(x)(y)(\llbracket \text { Mary }) \\
& =\exists x . \text { ate }(x)(\llbracket \text { Mary } \rrbracket)
\end{aligned}
$$

The second step is due to $*_{e}=*_{e}$.

Figure 2: $\llbracket$ Mary ate $\rrbracket$

and requiring the object of ate to be of that subtype (see Ben-Avi and Francez (2004)).

Is $*_{e}$ of type $e_{a d d i b l e}$ ? One way out is to include separate filler values for each sub-type of $e$, e.g., $*_{e_{\text {addible }}}$. But this cause a proliferation of those intelligible values as more selectional restrictions are imposed, for example, admitting

$$
\text { every girl smiled }
$$

but excluding

$$
\text { every chair smiled }
$$

by introducing a sub-type $e_{\text {animate }}$ of $e$ and requiring the subject of smile to be of this sub-type..

Populating models with unintelligible entities is typical to MTS in general. One of the main advantages of PTS is avoiding models an unintelligible entities with unclear ontological commitments, dealing only with syntactic entities, artefacts of meaning-conferring proof-systems.

\section{A Proof-Theoretic Semantics for a fragment of English}

\subsection{The language fragment}

I briefly present in this section the core of a fragment $E_{0}^{+}$of English. The core fragment consists of sentences with intransitive and transitive verbs, and determiner phrases with a (count, singular) noun and a determiner. For simplicity, I consider here only the positive determiners every and some. The inclusion of negative determiners such as no or at most three involve a certain complication (see Francez and Ben-Avi (2015)) that is orthogonal to the current issue. In addition, there is the copula isa. For technical reasons (made clear in Francez and Dyckhoff (2010)) I avoid the use of proper names, and use quantified subjects instead. Some typical sentences are listed below.

$$
\text { every/some girl smiles }
$$

$$
\text { every/some girl loves every/some boy }
$$

A typical sentence with an unexpressed argument to be considered is

$$
\text { every girl ate }
$$

I refer to expressions such as every girl, some boy as determiner phrases ( $d p \mathrm{~s})$.

\subsection{The meaning-conferring proof-system}

The PTS is based on a meaning-conferring naturaldeduction-proof system $N_{0}^{+}$with introduction rules and elimination rules ( $I / E$-rules) (see Figure $3)$. The proof-system is formulated over the language $L_{0}^{+}$, slightly extending $E_{0}^{+}$. Meta-variables 
$X$ schematise nouns, $P$ - intransitive verbs and $R$ - transitive verbs. Meta-variable $S$ ranges over sentences, and boldface lower-case $\mathbf{j}$, $\mathbf{k}$, etc., range over $\mathcal{P}$, a denumerable set of (individual) parameters, artefacts of the proof-system (not used to make assertions). Syntactically, a parameter in $L_{0}^{+}$ is also regarded as a $d p$. If a parameter occurs in $S$ in some position, I refer to $S$ as a pseudosentence, and if all $d p$ s in $S$ are parameters, the pseudo-sentence $S$ is ground. The ground pseudosentences play the role of atomic sentences, and their meaning is assumed given, externally to the ND proof-system.

The original full system takes into account also relative clauses, due to presence of which some scope-related indexing is essential, as there may be more than just two scope levels. For the purpose of dealing with unexpressed arguments, it is enough to have simple sentences, with just two scope-levels, for the subject and object $d p$ s. Furthermore, the general sentential variable $S$ can be instantiated by $P(-), R(-,-)$ and $R^{u}(-)$ for simple sentences headed by intransitive, transitive verbs and transitive verbs allowing intransitive use (lexically specified), respectively. Accordingly, I consider here a simplification of the original proof-system, not dealing with quantifier scope ambiguity. For any $d p$-expression $D$ having a quantifier, I use the notation $S[(D)]$ to refer to a sentence $S$ having a designated position filled by $D$.

For example, $S[$ (every $X)]$ refers to a sentence $S$ with a designated occurrence of every $X$. I use the conventions that within a rule, both $S\left[D_{1}\right], S\left[D_{2}\right]$ refer to the same designated position in $S$. I use $\Gamma, S$ for the context extending $\Gamma$ with sentence $S$.

Derivations (tree shaped), ranged over by $\mathcal{D}$ (possibly indexed) are defined recursively as usual. When presenting example derivations, the context $\Gamma$ is left implicit, and the notation $[\cdots]_{i}$ indicates an assumption discharged by an application of a rule.

The following is a convenient derived E-rule, that can be used to shorten derivations.

$$
\frac{\Gamma: S[(\text { every } X)] \quad \Gamma: \mathbf{j} \text { isa } X}{\Gamma: S[\mathbf{j}]}(e \hat{E})
$$

For example,

$$
\frac{\Gamma \text { : every girl smiled } \Gamma: \mathbf{j} \text { isa girl }}{\Gamma: \mathbf{j} \text { smiled }}(e \hat{E})
$$

\subsection{Canonical derivations and meaning}

Consider an arbitrary natural-deduction proofsystem intended as being meaning-conferring for the operators in some object language.

Definition 3.1 An $\mathcal{N}$-derivation $\mathcal{D}$ for $\vdash_{\mathcal{N}} \Gamma: \psi$ (for a compound $\psi$ ) is canonical iff it satisfies one of the following two conditions.

- The last rule applied in $\mathcal{D}$ is an I-rule (for the main operator of $\psi$ ).

- The last rule applied in $\mathcal{D}$ is an assumptiondischarging E-rule $e^{3}$ the major premise of which is some $\varphi$ in $\Gamma$, and its encompassed sub-derivations $\mathcal{D}_{1}, \cdots, \mathcal{D}_{n}$ (of the minor premises) are all canonical derivations of $\psi$.

- Denote by $\llbracket S \rrbracket_{\Gamma}^{c}$ the (possibly empty) collection of canonical derivations of $S$ from $\Gamma$.

For $\Gamma$ empty, the definition reduces to that of a traditional canonical proof. Note the recursion involved in this definition. The important observation regarding this recursion is that it always terminates via the first clause, namely by an application of an $I$-rule. I refer to such an application of an $I$-rule (ending a recursive path in the definition) as an essential application, the outcome of which is propagated throughout the canonical derivation by applications of the assumption-discharging $E$ rules. Note that each sub-derivation $\mathcal{D}_{i}$ may start with an essential application of an $I$-rule, thus having "parallel" essential applications of that rule. I refer to a sequence of occurrences of $\psi$ in a canonical $\mathcal{D}$ starting in the conclusion of an essential application of an $I$-rule and ending in the conclusion of $\mathcal{D}$ as a propagation chain of $\psi$ in $\mathcal{D}$.

The major observation here is that the conclusion $\psi$ of a canonical derivation $\mathcal{D}$ from open assumptions, ending a propagation chain, was:

1. inferred (possibly more than once) by the $e s$ sential applications of the $I$-rule of its main operator, and

2. propagated through applications of assumption-discharging $E$-rules ${ }^{4}$ to its position as the final conclusion of $\mathcal{D}$.

\footnotetext{
${ }^{3}$ General elimination rules (GE) have this structure, hence their association with permuting conversions during normalisation. See more on this in Negri and von Plato (2001).

${ }^{4}$ In propositional intuitionistic logic, say with Gentzen's $N J$ ND-system, this happens with disjunction.
} 


$$
\begin{gathered}
\overline{\Gamma, S: S}(A x) \\
\frac{\Gamma, \mathbf{j} \text { isa } X: S[\mathbf{j}]}{\Gamma: S[(\text { every } X)]}(e I) \quad \frac{\Gamma: \mathbf{j} \text { isa } X \quad \Gamma: S[\mathbf{j}]}{\Gamma \vdash S[(\text { some } X)]}(s I) \\
\frac{\Gamma: S[(\text { every } X)] \quad \Gamma: \mathbf{j} \text { isa } X \quad \Gamma, S[\mathbf{j}]: S^{\prime}}{\Gamma: S^{\prime}}(e E) \quad \frac{\Gamma: S[(\text { some } X)] \quad \Gamma, \mathbf{j} \text { isa } X, S[\mathbf{j}]: S^{\prime}}{\Gamma: S^{\prime}}(s E) \\
\text { where } \mathbf{j} \text { is fresh in }(e I), \text { and }(s E) .
\end{gathered}
$$

Figure 3: The simplified rules for $N_{0}^{+}$

The (reified) meaning of a sentence $S$ (in the considered object language), denoted by $\llbracket S \rrbracket$, is defined by

\section{Definition 3.2 (reified meaning)}

$$
\llbracket S \rrbracket=d f \cdot \lambda \Gamma \cdot \llbracket S \rrbracket_{\Gamma}^{c}
$$

This is a very fine-granularity notion of meaning, see Francez (2014). A natural coarsening is obtained by considering grounds of assertion.

\section{Definition 3.3 (grounds for assertion)}

$$
G A \llbracket S \rrbracket=\left\{\Gamma \mid \vdash^{c} \Gamma: S\right\}
$$

Thus, any $\Gamma$ that canonically derives $S$ serves as grounds for assertion of $S$. This is naturally extended to $G A \llbracket \Gamma \rrbracket$, the grounds of asserting all $S^{\prime} \in \Gamma$. For the methodological role of this concept in the theory of meaning adhered to by PTS, see Dummett (1993).

By appealing to equality of grounds, we get an equivalence relation coarser than meaning as defined above, inducing a natural 'sameness of meaning' relation.

Definition 3.4 (equi-groundedness) $S_{1}$ and $S_{2}$ are equi-grounded, denoted by $S_{1} \equiv_{G A} S_{2}$, iff $G A \llbracket S_{1} \rrbracket=G A \llbracket S_{2} \rrbracket$.

Grounds of assertion lead (see Francez (2015a)) to the following notion of proof-theoretic consequence, that captures the pre-theoretic entailment.

\section{Definition 3.5 (proof-theoretic consequences)}

$S$ is a proof-theoretic consequence of $\Gamma(\Gamma \Vdash S)$ iff $G A \llbracket \Gamma \rrbracket \subseteq G A \llbracket S \rrbracket$.

Thus, proof-theoretic consequences is based on grounds propagation: every grounds for collectively asserting all of $\Gamma$ are already grounds for asserting $S$.

\section{Proof-theoretic semantics for unexpressed arguments}

In this section, I extend the proof-system with $I / E$-rules for the sentences allowing (lexically licensed) intransitive uses of transitive verbs, underlying the proof-theoretic account of their meaning.

The main ideas on which the PTS for implicit arguments is based is the following:

- Sameness of meaning is based on sameness of I-rules, implying sameness of canonical derivations.

- The reduction of an argument is reflected proof-theoretically by omission of a premise (in I-rules).

\subsection{Adding something}

As the alert reader might have noticed, all the $n p \mathrm{~s}$ in $E_{0}^{+}$contain an explicit noun-component; for example, some girl, every boy. I will refer to such nps as nominally qualified. Indeed, typical NL-quantification is what is known as restricted quantification. However, for the sake of the specification of the meaning of sentences with an implicit argument, what is needed ${ }^{5}$ is to augment the language fragment with an $n p$ expressing $u n$ restricted existential quantification in the form of something, lacking a nominal qualification.

The nominally-unqualified existential quantification in object position is governed by the fol-

\footnotetext{
${ }^{5}$ Another alternative is to consider a universal noun thing, and use it as the noun-qualifier. I consider this less elegant in the context of PTS.
} 
lowing $I / E$-rules ${ }^{6}$.

$$
\begin{gathered}
\frac{\Gamma: \mathbf{j} R \mathbf{k}}{\Gamma: \mathbf{j} R \text { something }}(u s I) \\
\frac{\Gamma: \mathbf{j} R \text { something } \Gamma, \mathbf{j} R \mathbf{k}: S^{\prime}}{\Gamma: S^{\prime}}(u s E) \quad \mathbf{k} \text { fresh. }
\end{gathered}
$$

The difference between those two $I / E$-rules and the $I / E$-rules for nominally-qualified $n p$ s $((s I)$ and $(s E)$ below) is the absence of a nominallyqualifying premise $\mathbf{k}$ isa $X$ (for some noun $X$ ) in the $I$-rule, and the absence of a corresponding discharged assumption in the $E$-rule.

For example,

$$
\begin{gathered}
\frac{\Gamma: \mathbf{j} \text { ate } \mathbf{k}}{\Gamma: \mathbf{j} \text { ate something }}(u s I) \\
\frac{\Gamma: \mathbf{j} \text { ate something } \Gamma, \mathbf{j} \text { ate } \mathbf{k}: S^{\prime}}{\Gamma: S^{\prime}}(u s E)
\end{gathered}
$$

\subsection{Sentential meanings}

In contrast to the view of argument reduction (mentioned above), I present separate I-rules for the transitive and intransitive uses of sentences with $R^{u}$-verbs; furthermore, the same $I$ rule serves both for the introduction for sentences with $R^{u}$-verbs with an unexpressed object and for the corresponding $R$ with an explicit object something. This establishes the sameness of meaning of pairs of sentences like (1(ii)) and (2), where this sameness of meaning is based on both sentences having the same grounds of assertion.

\section{Transitive verb with an expressed object:}

These are the usual rules $(s I)$ and $(s E)$, simplified to objects only, of simple sentences.

$$
\begin{gathered}
\frac{\Gamma: \mathbf{k} \text { isa } X \quad \Gamma: \mathbf{j} R \mathbf{k}}{\Gamma: \mathbf{j} R \text { some } X}(s I) \\
\frac{\Gamma: \mathbf{j} R \text { some } X \quad \Gamma, \mathbf{k} \text { isa } X, \mathbf{j} R \mathbf{k}: S^{\prime}}{\Gamma: S^{\prime}}(s E) \\
\mathbf{k} \text { fresh }
\end{gathered}
$$

\footnotetext{
${ }^{6}$ Similar rules govern something in a subject position, but they do not matter here and are omitted.
}

\section{Transitive verb with an unexpressed object:}

$$
\begin{gathered}
\frac{\Gamma: \mathbf{j} R^{u} \mathbf{k}}{\Gamma: \mathbf{j} R^{u}}(u I) \\
\frac{\Gamma: \mathbf{j} R^{u} \Gamma_{\mathbf{j}} R^{u} \mathbf{k}: S^{\prime}}{\Gamma: S^{\prime}}(u E)_{\mathbf{k} \text { fresh }}
\end{gathered}
$$

Here only a sentence with an $R^{u}$-verb can be used. Note the "missing" premise $\mathbf{k}$ isa $X$ (for some $X$ ), leaving the object nominallyunqualified, hence omit-able. It is crucial to note that the subject of $R^{u}$ has to be a parameter, assuring the meaning-identity with the explicit existentially case below. The parameter $\mathbf{j}$ is a basis for introduction of a universally quantified subject with a higher scoping quantifier.

For example, with $R^{u}=$ ate (with contexts omitted):

$\frac{\mathbf{j} \text { ate } \mathbf{k}}{\mathbf{j} \text { ate }}(u I) \quad \frac{\mathbf{j} \text { ate } \mathbf{j} \text { ate } \mathbf{k}: S^{\prime}}{\Gamma: S^{\prime}}(u E) \mathbf{k}$ fresh

Note that no similar $(u I)$-application can be used to derive, for example, $\mathbf{j}$ smiled, under the assumption that smiled is a proper intransitive verb.

Example 4.2 As an example, I show that

$\vdash$ every girl ate some apple : every girl ate

The derivation is shown in Figure 4. So, the "essence" of the derivation is not using the premise $\mathbf{k}$ isa apple, later discharged by the application of $(s E)$, in deriving $\mathbf{j}$ ate.

\subsection{Sameness of meaning}

The following proposition, expressing the equality of meaning between intransitive $R^{u}$-headed pseudo-sentence and explicitly existentiallyquantified $R$-headed pseudo-sentences is a simple consequence of those rules.

\section{Proposition 4.1}

$$
\llbracket \mathbf{j} R^{u} \rrbracket=\llbracket \mathbf{j} R \text { something } \rrbracket
$$

and in particular

$$
\mathbf{j} R^{u} \equiv_{G A} \mathbf{j} R \text { something }
$$

Proof: immediate, as there is a one-one correspondence between canonical derivations on both 


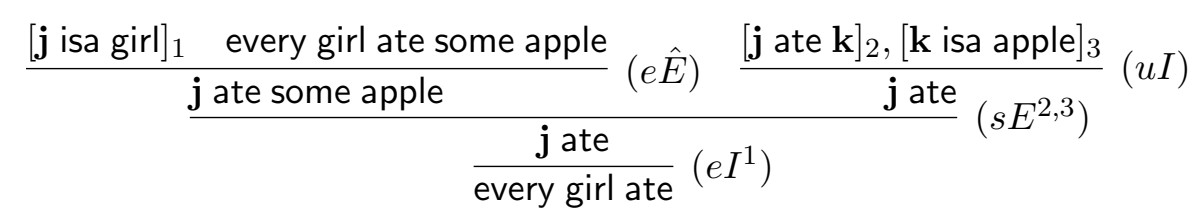

Figure 4: Derivation of every girl ate

sides of the equality, because the premises of the respective $I$-rules are the same.

A consequence of proposition 4.1 is the following theorem ${ }^{7}$, establishing that the sameness of meaning for ground pseudo-sentences with $R^{u}$ verbs extends to a congruence of sameness of meaning for arbitrary sentences with $R^{u}$-verbs in the fragment (namely, arbitrary quantified $n p$ s as subject). As we have here only two possible subjects, we state the theorem for both, explicitly.

\section{Theorem 4.1}

$$
\llbracket \text { every } X R^{u} \rrbracket=\llbracket \text { every } X \quad R \text { something } \rrbracket
$$

$$
\text { 【some } X R^{u} \rrbracket=\llbracket \text { some } X \quad R \text { something } \rrbracket
$$

Proof: Suppose $\mathcal{D}$ is a canonical derivation of every $X R^{u}$. Then, omitting contexts, $\mathcal{D}$ has to have a subderivation of the following form.

$$
\begin{aligned}
& {[\mathbf{j} \text { isa } X]_{i}} \\
& \mathcal{D}^{\prime} \\
& \frac{\mathbf{j} R^{u}}{\text { every } X R^{u}}\left(e I^{i}\right)
\end{aligned}
$$

There are two sub-cases to consider, depending whether $\mathcal{D}^{\prime}$ itself is canonical or not.

1. First, assume $\mathcal{D}^{\prime}$ is canonical. By proposition $4.1, \mathcal{D}^{\prime}$ is also a canonical derivation of

$$
\begin{gathered}
{[\mathbf{j} \text { isa } X]^{i}} \\
\mathcal{D}^{\prime} \\
\mathbf{j} R \text { something }
\end{gathered}
$$

And, hence,

$$
\begin{gathered}
{[\mathbf{j} \text { isa } X]_{i}} \\
\mathcal{D}^{\prime} \\
\frac{\mathbf{j} R \text { something }}{\text { every } X R \text { something }}\left(e I^{i}\right)
\end{gathered}
$$

\footnotetext{
${ }^{7}$ Note that neither Blom et al. (2012) nor Giorgolo and Asudeh (2012) relate to this issue, discussing only sentences with proper names as subjects.
}

is a canonical derivation, establishing

$$
\text { 【every } X R^{u} \rrbracket \subseteq \llbracket \text { every } X \quad R \text { something } \rrbracket
$$

Showing

$$
\llbracket \text { every } X \quad R \text { something } \rrbracket \subseteq \llbracket \llbracket \text { every } X R^{u} \rrbracket
$$

is similar.

2. Next, suppose $\mathcal{D}^{\prime}$ is not canonical. Thus, the conclusion $\mathbf{j} R^{u}$ was obtained by an application of an $E$-rule. In the small fragment considered here, there are two possible $E$-rules that could yield this consequence.

(a) The one possibility is the $(e \hat{E})$-rule. This means that $\mathcal{D}^{\prime}$ must have the following form.

$$
\frac{\begin{array}{c}
\mathcal{D}_{1}^{\prime} \\
\text { every } Y R^{u}
\end{array} \mathcal{D}_{2}^{\prime} \mathbf{j} \text { isa } Y}{\mathbf{j} R^{u}}\left(e \hat{E}^{j}\right)
$$

In the subderivation $\mathcal{D}_{1}^{\prime}$, the conclusion must have been introduced by the $(e I)$ rule. So this premise looks like

$$
\begin{aligned}
& {[\mathbf{k} \text { isa } Y]_{j}} \\
& \mathcal{D}_{1}^{\prime \prime} \\
& \frac{\mathbf{k} R^{u}}{\text { every } Y R^{u}}\left(e I^{j}\right)
\end{aligned}
$$

Since $\mathcal{D}_{1}^{\prime \prime}$ is smaller than $\mathcal{D}$, by induction it follows that

$$
\begin{gathered}
{[\mathbf{k} \text { isa } Y]_{j}} \\
\mathcal{D}_{1}^{\prime \prime} \\
\frac{\mathbf{k} R \text { something }}{\text { every } Y R \text { something }}\left(e I^{j}\right)
\end{gathered}
$$

from which we get the required derivation

$$
\begin{aligned}
& \mathcal{D}_{1}^{\prime} \quad \mathcal{D}_{2}^{\prime} \\
& \frac{\text { every } Y R \text { something } \mathbf{j} \text { isa } Y}{\mathbf{j} R \text { something }}\left(e \hat{E}^{j}\right)
\end{aligned}
$$

The other direction is similar. 
(b) The other possibility is that the relevant $E$-rules are $(s E)$ and $(u E)$ (for a subject some $X)$. The details are similar to the previous case and omitted.

This proof could be extended for subjects with other determiners, e.g. at least seven girls ate, having the same meaning as at least seven girls ate something, not in the current fragment, though.

There is an interesting observation arising from the proof-theoretic meaning device above. Consider sentences with intensional transitive verb (ITVs) with an indefinite object $n p$, like

$$
\text { Mary seeks/looks for a secretary }
$$

Such sentences are known to have a notional reading (unspecific) of their object. Thus, (29) has a reading by which Mary looks for any secretary.

Such sentences have no equivalent counterpart with an unexpressed object.

$$
(*) \text { Mary seeks/looks for }
$$

The explanation for the impossibility of the object omission can be found in the proof-theoretic semantics for ITVs provided in Francez (2016). Under this semantics, the objects of ITVs are different kind of parameters than the individual parameters, for which the omission rules present here do not apply. This shows the power of syntactic methods, introducing formal distinctions not carrying any ontological commitments, unlike the need to populate models with entities of unclear nature as in model-theoretic semantics.

\section{Conclusions}

In this paper I have presented a proof-theoretic semantics for sentences headed by transitive verbs allowing an unexpressed (implicit) object. Such sentences are shown to have the same (prooftheoretic) meaning as the same sentences with an explicit existentially quantified object something.

This semantics is contrasted with a modeltheoretic semantics based on truth-conditions in models. The models used contain in their domain "filler" elements, that have an unclear extratheoretic significance with an unclear ontological commitments. In contrast, the proof-theoretic meaning is appealing to formal (syntactic) resources that carry no ontological commitment.
Furthermore, the sameness of meaning is based on sameness of deductive role within a meaningconferring proof-system, based on use.

\section{References}

Gilad Ben-Avi and Nissim Francez. 2004. Categorial grammar with ontology-refined types. In Categorial Grammars 2004: An efficient tool for Natural Language Processing. Montpellier, France.

Chris Blom, Philippe de Groote, Yoad Winter, and Joost Zwarts. 2012. Implicit arguments: event modification or option type categories. In Maria Aloni, Vadim Kimmelman, Floris Roelofsen, Galit W. Sasson, Katrin Schulz, and Matthijs Westera, editors, Proceedings of the 2011 Amsterdam Colloquium. Springer, LNCS 7218, pages 240-2501.

Greg N. Carlson. 1984. Thematic roles and their role in semantic interpretation. Linguistics 22:259- 279.

David Dowty. 1982. Grammatical relations and montague grammar. In Pauline I. Jacobson and Geoffrey K. Pullum, editors, The Nature of Syntactic Representation, Reidel, Dordrecht, pages 79-130.

Michael Dummett. 1993. The Logical Basis of Metaphysics. Harvard University Press, Cambridge, MA., paperback edition. Hardcover 1991.

Nissim Francez. 2014. The granularity of meaning in proof-theoretic semantics. In Nicholas Asher and Sergei Soloview, editors, Proceedings of the 8th International Conference on Logical Aspects of Computational Linguistics (LACL), Toulouse, France, June 2014. Springer Verlag, LNCS 8535, Berlin, Heidelberg, pages 96-106.

Nissim Francez. 2015a. On distinguishing prooftheoretic consequence from derivability. Logique et Analysis (to appear).

Nissim Francez. 2015b. Proof-theoretic Semantics. College Publications, London.

Nissim Francez. 2016. Proof-theoretic semantics for intensional transitive verbs. Journal of Semantics 33(4):803-826. Doi: 10.1093/jos/ffv013.

Nissim Francez and Gilad Ben-Avi. 2015. A proof-theoretic reconstruction of generalized quantifiers. Journal of Semantics 32(3):313-371. Doi:10.1093/jos/ffu001.

Nissim Francez and Roy Dyckhoff. 2010. Prooftheoretic semantics for a natural language fragment. Linguistics and Philosophy 33(6):447-477.

Gianluca Giorgolo and Ash Asudeh. 2012. Missing resources in a resource-sensitive semantics. In Proceedings of the 17th Lexical Functional Grammar (LFG) conference, Bali, June-July 2012. CSLI online publications. 
Sara Negri and Jan von Plato. 2001. Structural Proof

Theory. Cambridge University Press, Cambridge, UK. 